\title{
Breath-by-Breath Analysis of Cardiorespiratory Interaction for Quantifying Developmental Maturity in Premature Infants
}

\author{
Matthew T. Clark \\ Craig G. Rusin \\ John L. Hudson \\ Hoshik Lee \\ William \& Mary \\ John B. Delos \\ William \& Mary, jbdelos@wm.edu
}

See next page for additional authors

Follow this and additional works at: https://scholarworks.wm.edu/aspubs

Part of the Physics Commons

\section{Recommended Citation}

Clark, M. T., Rusin, C. G., Hudson, J. L., Lee, H., Delos, J. B., Guin, L. E., ... \& Moorman, J. R. (2011). Breathby-breath analysis of cardiorespiratory interaction for quantifying developmental maturity in premature infants. Journal of Applied Physiology, 112(5), 859-867.

This Article is brought to you for free and open access by the Arts and Sciences at W\&M ScholarWorks. It has been accepted for inclusion in Arts \& Sciences Articles by an authorized administrator of W\&M ScholarWorks. For more information, please contact scholarworks@wm.edu. 
Authors

Matthew T. Clark, Craig G. Rusin, John L. Hudson, Hoshik Lee, John B. Delos, and et al. 


\title{
Breath-by-breath analysis of cardiorespiratory interaction for quantifying developmental maturity in premature infants
}

\author{
Matthew T. Clark, ${ }^{1}$ Craig G. Rusin, ${ }^{2}$ John L. Hudson, ${ }^{1}$ Hoshik Lee, ${ }^{3}$ John B. Delos, ${ }^{3}$ Lauren E. Guin, ${ }^{2}$ \\ Brooke D. Vergales, ${ }^{4}$ Alix Paget-Brown, ${ }^{4}$ John Kattwinkel, ${ }^{4}$ Douglas E. Lake, ${ }^{2,5}$ and J. Randall Moorman ${ }^{2}$ \\ ${ }^{1}$ Department of Chemical Engineering, University of Virginia, Charlottesville; ${ }^{2}$ Department of Medicine, Division of \\ Cardiovascular Medicine, University of Virginia Health System, Charlottesville; ${ }^{3}$ Department of Physics, College of William \\ and Mary, Williamsburg; ${ }^{4}$ Department of Pediatrics, Division of Neonatology, University of Virginia Health System, \\ Charlottesville; and ${ }^{5}$ Department of Statistics, University of Virginia, Charlottesville, Virginia
}

Submitted 13 September 2011; accepted in final form 14 December 2011

Clark MT, Rusin CG, Hudson JL, Lee H, Delos JB, Guin LE, Vergales BD, Paget-Brown A, Kattwinkel J, Lake DE, Moorman JR. Breath-by-breath analysis of cardiorespiratory interaction for quantifying developmental maturity in premature infants. $J$ Appl Physiol 112: 859-867, 2012. First published December 15, 2011; doi:10.1152/japplphysiol.01152.2011.--In healthy neonates, connections between the heart and lungs through brain stem chemosensory pathways and the autonomic nervous system result in cardiorespiratory synchronization. This interdependence between cardiac and respiratory dynamics can be difficult to measure because of intermittent signal quality in intensive care settings and variability of heart and breathing rates. We employed a phase-based measure suggested by Schäfer and coworkers (Schäfer C, Rosenblum MG, Kurths J, Abel HH. Nature 392: 239-240, 1998) to obtain a breath-by-breath analysis of cardiorespiratory interaction. This measure of cardiorespiratory interaction does not distinguish between cardiac control of respiration associated with cardioventilatory coupling and respiratory influences on the heart rate associated with respiratory sinus arrhythmia. We calculated, in sliding 4-min windows, the probability density of heartbeats as a function of the concurrent phase of the respiratory cycle. Probability density functions whose Shannon entropy had a $<0.1 \%$ chance of occurring from random numbers were classified as exhibiting interaction. In this way, we analyzed 18 infant-years of data from 1,202 patients in the Neonatal Intensive Care Unit at University of Virginia. We found evidence of interaction in 3.3 patient-years of data $(18 \%)$. Cardiorespiratory interaction increased several-fold with postnatal development, but, surprisingly, the rate of increase was not affected by gestational age at birth. We find evidence for moderate correspondence between this measure of cardiorespiratory interaction and cardioventilatory coupling and no evidence for respiratory sinus arrhythmia, leading to the need for further investigation of the underlying mechanism. Such continuous measures of physiological interaction may serve to gauge developmental maturity in neonatal intensive care patients and prove useful in decisions about incipient illness and about hospital discharge.

preterm infants; synchronization; intensive care; postnatal development; cardioventilatory coupling; respiratory sinus arrhythmia

A CURRENT CONCEPT OF HEALTH is that organs couple with one another through extracellular signaling or the nervous system. Illnesses such as systemic inflammation disrupt this complex, finely adaptive system and lead to uncoupling (17). When the organs have obvious oscillatory dynamics as the heart and the lungs do, presence of coupling is taken as a sign of good health. The cardiorespiratory interaction is mediated through complex central and autonomic nervous system mechanisms. Conspic-

Address for reprint requests and other correspondence: M. T. Clark, 102 Engineers' Way, PO Box 400741, Charlottesville, VA 22904-4741. uous examples of such interaction are respiratory sinus arrhythmia and cardioventilatory coupling. Respiratory sinus arrhythmia (RSA) is the familiar rise and fall of heart rate with respiration $(10,18,27,42)$, mediated by the baroreflex, respiratory gating, or both $(9,11,26)$. Presence of RSA correlates with good outcome in a number of clinical situations, including intensive care $(1,37,39)$. Cardioventilatory coupling (CVC) is the preferential synchronization of inhalation at integer ratios with the heartbeat, mediated by baroreceptor inputs to the respiratory pattern generator (52). The presence of CVC has been documented in neurologically healthy adults in sleeping states and under anesthesia $(16,33,51)$.

Coupling of heart rate and ventilation is present in healthy infants born at term $(23)$, infants born preterm $(3,25)$, and even in late-term fetuses (19). Respiratory control of cardiac dynamics has been shown to increase with postnatal age in anesthetized term piglets (24). Here, we study continuous changes in cardiorespiratory interaction in waking term and preterm infants. Late gestation is a busy time with regard to development of central nervous system interconnections that reside between and within the brain stem and limbic system and underlie cardiorespiratory interaction (6). The complex integration of signaling processes and receptor expression proceeds in a presumably orderly but, to the outside observer, highly nonlinear way, especially when major portions of the development take place postnatally in the premature infant in the Neonatal Intensive Care Unit (NICU). These concepts are of clinical relevance in bedside neonatology. Determining an acceptable level of physiological maturity is an important task assigned to the clinician caring for the NICU patient who was born preterm. For example, nearly all infants born very preterm $(<1,500 \mathrm{~g}$ birth weight) will have at least one life-threatening apneic event during their initial hospitalization. Clinicians caring for such babies are challenged with deciding when the infant has sufficient physiological maturity to be discharged safely into an environment lacking the intensive and continuous cardiorespiratory monitoring available in the NICU setting (7). Mature physiological cardiorespiratory interaction might not be just a matter of waiting until a consensus postmenstrual age, since fully functional physiological maturity of respiratory control comes later and later the more prematurely an infant is born $(7,13)$. The presence of apparent life-threatening apneic events (5) and SIDS (28) in the first several months after birth in premature infants suggests that, for some, physiological maturity comes late if at all.

Although the degree of cardiorespiratory interaction might provide information about the maturation process, it is hard to 
Table 1. Demographic information for infants in the University of Virginia Neonatal Intensive Care Unit from January 2009 to June 2011

\begin{tabular}{lrlcr}
\hline \hline \multicolumn{1}{c}{ Population } & \multicolumn{1}{c}{$N$} & \multicolumn{1}{c}{ Birth Weight,* g } & Gestational Age,* wk & Males \\
\hline All & 1,202 & $2,339(1,615)$ & $35(7)$ & 644 \\
Non-VLBW & 910 & $2,705(1,120)$ & $37(5)$ & 493 \\
VLBW & 292 & $1,010(500)$ & $27(4)$ & 151 \\
Discharge home (VLBW) & 148 & $1,140(494)$ & $28.5(4.5)$ & 79 \\
\hline
\end{tabular}

VLBW, very low birth weight. *Birth weight and gestational age values are medians (interquartile ranges).

measure. Variations in breathing frequency and depth, and in the lag between ventilation and heart rate can confound temporal and frequency domain analysis of cardiorespiratory interaction in infants (21-23). Confident detection of cardiorespiratory interaction requires high-quality EKG and chest impedance signals. The long, uninterrupted time series data required to achieve this using conventional time- and frequency-domain analysis are not common enough to permit study of cardiorespiratory interaction in the NICU. Another approach is to use techniques that are insensitive to missing data. One such approach is to identify individual beats and breaths only when the data allow, and Schäfer and coworkers gave an example of this approach in a study of cardiorespiratory interaction in young adults (46). In the present work, we followed their technique for determining the probability of heartbeats as a function of respiratory phase as a measure of cardiorespiratory interaction. The fundamental assumption is that differential distribution of heartbeats within the respiratory cycle is evidence for cardiorespiratory interaction.

Cardiorespiratory interaction, even when measured well, is only intermittent at best. In eight young adults carefully studied at rest, Schäfer and coworkers found evidence for coupling for only $\sim 30 \mathrm{~min}$ of the $4 \mathrm{~h}$ studied. In infants, it is, for example, more prevalent during quiet sleep than REM sleep $(20,21)$. Our major hypothesis is that measuring cardiorespiratory interaction in premature infants may be clinically useful in assessing the developmental maturity of physiological coupling. To begin to test this idea, we have measured cardiorespiratory interaction in a large population of consecutively admitted infants in a tertiary care NICU. To this end, we implemented phase-based measures (46) in an effort to quantify the effect of breathing on the heartbeat, the predominant direction for cardiorespiratory interaction in infants (38). We present our methods for automatically analyzing continuous physiological waveforms and the relation between this measure of coupling and developmental maturity, and discussion of the underlying mechanisms.

\section{METHODS}

Ethical approval. The University of Virginia (UVA) Institutional Review Board gave permission for this study. This is an observational study, and no experiments were conducted on humans.

Patient population. We collected cardiorespiratory waveforms continuously from the bedside monitors of 1,202 infants admitted consecutively to the UVA NICU from January, 2009 through June, 2011. Patient demographic data, including admission and discharge dates, types and times of respiratory support, and disposition and status at discharge were obtained from the clinical records of 292 very low birth weight (VLBW; $<1,500 \mathrm{~g}$ at birth) infants.
Of these, 148 were discharged to home without any respiratory support, including supplemental oxygen. These infants were therefore considered to have recovered from all acute illnesses encountered during the study period and had been judged by the attending physician to have adequate cardiorespiratory stability to be discharged from the hospital without cardiorespiratory monitoring. Analysis of waveforms from VLBW patients was restricted to times without endotracheal intubation, since mechanical ventilation may alter the relationship between respiratory and cardiac control. Estimated gestational age (EGA) was calculated as the time from the date of onset of the mother's last menses to the date of birth. Post menstrual age (PMA) was calculated as the time from the date of onset of the mother's last menses to the time of analysis, regardless of the date of birth. Table 1 shows demographic information for the study population. Figure 1 shows a schematic of the UVA NICU dataset. This real-world clinical dataset was limited by absence and poor quality of chest impedance. Nonetheless, 3.3 patient-years of cardiorespiratory interaction were found. Results are shown for non-VLBW infants, extubated VLBW infants, and intubated VLBW infants.

Data acquisition. We monitored infants in the NICU with GE bedside monitors using three electrocardiogram (EKG) leads digitized at $240 \mathrm{~Hz}$. A small-amplitude and high-frequency voltage is applied between two of the EKG leads. The chest impedance pneumograph is the applied voltage divided by the measured current, and was digitized at $60 \mathrm{~Hz}$. A central network server (BedMasterEx, Excel Medical, Jupiter, FL) behind a clinical firewall collected these data and transmitted them to a customized parallel storage and computing cluster behind an additional firewall. Data were analyzed in parallel on a 10-computer cluster with 80 processors, $40 \mathrm{~GB}$ of RAM, and $100 \mathrm{~TB}$ of hard disk space. A total of 60.7 patient-years of data (4 TB) were collected from bedside monitors of 1,202 infants in the University of Virginia NICU. Waveforms were analyzed in three steps: noise quantification, breath and QRS detection, and measurement of cardiorespiratory interaction.

Waveform analysis: noise quantification. Our measurement of cardiorespiratory interaction relies on automated detection algorithms that require minimal noise. We quantified noise levels by adding noise of clinically appropriate frequency content to noise-free waveforms and calculating signal quality indexes. Clinically appropriate noise was derived from an analysis of a large sample of clinical data from many infants. Signal quality indexes were analyzed as a function of added noise, and we restricted further analysis to signals with low estimated noise levels. Detailed discussion of signal quality measures for EKG and chest impedance time series are provided in the APPENDIX.

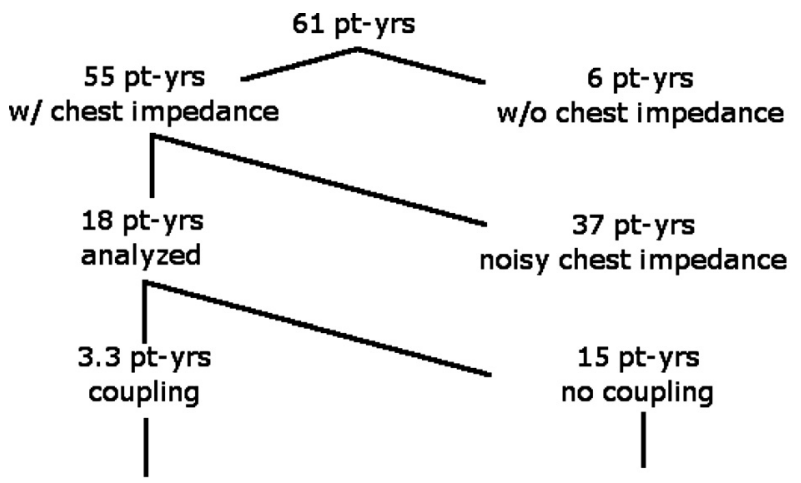

2.0 pt-yrs Non-VLBW

\section{2 pt-yrs extubated VLBW}

0.1 pt-yrs intubated VLBW
6.7 pt-yrs Non-VLBW

7.2 pt-yrs extubated VLBW 1.0 pt-yrs intubated VLBW
Fig. 1. Distribution of data among University of Virgina (UVA) neonatal intensive care unit (NICU) patients. 
Waveform analysis: breath and QRS detection. QRS complexes were automatically identified on the EKG lead with the lowest noise using three detection algorithms. One is threshold-based (40) as implemented by Clifford and coworkers $(35,50)$. The second removes the T- and P-waves by using a high-pass filter. QRS complex locations were defined as positive crossings of the $x$-axis in Hilbert space (30). The final method decomposed the EKG signal using a continuous Haar wavelet transform at scales $2^{n}$, where $n=1,2 \ldots 5$. The squared normalized wavelet coefficients were averaged, the root-mean-square over $80 \mathrm{~ms}$ was calculated, and $80-\mathrm{ms}$ segments were centered at peaks in the resulting signal collected. Segments that had significant correlation $(P<0.01)$ with at least $50 \%$ of other segments were defined as QRS complexes. Complexes detected by each method were represented with kernels of unit height and width of $80 \mathrm{~ms}$ at three standard deviations. The three kernelled time series were averaged, such that peaks identified by all three methods within the same $80-\mathrm{ms}$ window had minimum amplitude of 0.67 . We defined heartbeats as peaks exceeding the value 0.8 in the averaged signal based on visual inspection. The root mean squared difference between averaged heart rate from the monitor and our detection scheme was 1.8 beats/min.

Features of interest in the chest impedance pneumograph are times at which the lungs are full and empty. Cardiac artifact was removed from the signal using a heart time transform (34). Full and empty lung states were found using peak and trough detection on a zero-phase band-pass filtered signal. A high-frequency cutoff was defined using a discrete Haar wavelet transform of the chest impedance time series normalized to mean 0 and variance 1 . The variance was calculated at each scale, and the respiratory band was identified by collecting scales with the most variance until at least $87 \%$, or $\left(1-\mathrm{e}^{-2}\right)$, of the total variance was included. The high-frequency cutoff was defined by the largest scale below the respiratory band. The low-frequency cutoff was varied using MatLab (MathWorks, http://www.mathworks.com) implementations of golden section search and parabolic interpolation. The minimization criterion was the coefficient of variation for the Hilbert space amplitude. The minimization was bounded by frequencies corresponding to the length of the time series and the shortest scale above the respiratory band.

Waveform analysis: measurement of cardiorespiratory interaction. To quantify cardiac dependence on respiration, we studied the temporal association of heartbeats within the respiratory phase. Each heartbeat was assigned a value corresponding to the phase of respiration at which it occurred. The probability of a heartbeat was calculated as a function of concurrent respiratory phase using a kernel density estimate. Interaction of heart to lungs led to localization of heartbeats within the respiratory cycle that was evident in the probability densities. We created these probability density functions every $30 \mathrm{~s}$ for sliding windows of $4 \mathrm{~min}$. We used the Shannon entropy (48), $S$, of the probability density function to quantify the degree of localization of heartbeats in respiratory phase. Lower values of entropy denote more localization of heartbeats in a respiratory phase and, therefore, more coupling.

We define a measure of cardiorespiratory interaction, $C$, as the fraction of overlapping 4-min records in a 2-h period that had a $<0.1 \%$ probability of arising from noise. The minimum number of 4-min windows was 10 , with at least 30 heartbeats each. This corresponds to a minimum of 11-40 min every $2 \mathrm{~h}$.

\section{RESULTS}

Selection of data for analysis. Although 61 patient-years of data were collected, not all data were suitable for the analysis of cardiorespiratory interaction. Although the noise in the EKG signal was generally low enough for QRS detection, the chest impedance signal was of more variable quality. High-quality EKG data ( $<20 \%$ noise) were available in $99 \%$ of the dataset. Only 55 patient-years of the dataset had chest impedance recorded. We accepted chest impedance signals having noise commensurate with a sine wave plus $85 \%$ additional noise. We found that $33 \%$ of available data, corresponding to 18 patientyears, were of sufficiently low noise for analysis. We justified the inclusion of data with such a large apparent noise component because both normal respiratory variability and normal non-sinusoidal breathing are detected as noise in our analysis. The chest impedance in Fig. 2A, for example, has an estimated noise of $21 \%$.

An example of the analysis. Figure $2, A$ and $B$, shows two exemplary time series of EKG and chest impedance from the same infant. In Fig. 2A, alternating QRS complexes repeatedly occurred in mid-exhale and mid-inhale. In Fig. $2 B$, the timing of QRS complexes varied throughout the time series. The probability densities for heartbeats as a function of respiratory phase for 4-min records containing these time series are shown in Fig. $2 C$, showing that the timing of heartbeats depended on the respiratory state in Fig. $2 A$ but not in Fig. $2 B$. It is important to note that these two windows have comparable heart rate variability: 94.7 and 95.4 beats $/ \mathrm{min}^{2}$, respectively. This indi-
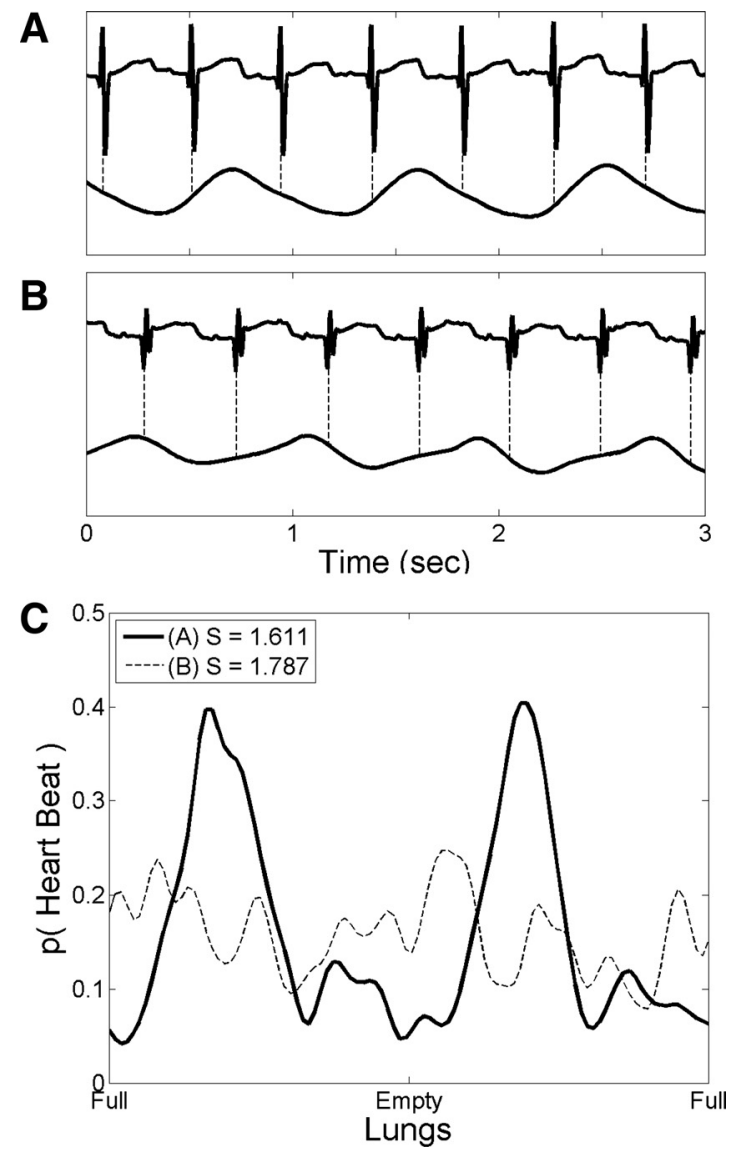

Fig. 2. A: exemplary electrocardiogram (EKG) and chest impedance signals showing cardiorespiratory interaction. Broken lines show that heartbeats repeatedly occur at the same phase of respiration. $B$ : exemplary EKG and chest impedance signals with no cardiorespiratory interaction. $C$ : probability density of heartbeats as a function of respiratory phase. The solid line is from a 4-min record containing the data in $A$ and shows the localization of heartbeats relative to the phase of respiration. The broken line is from a 4-min record containing the data in $B$ and shows an absence of cardiorespiratory interaction. The difference is quantified by S, the value of the Shannon entropy, which is lower at the time of cardiorespiratory interaction. The heart rate variance was very similar for both records $\left(94-96\right.$ beats $\left./ \mathrm{min}^{2}\right)$. 

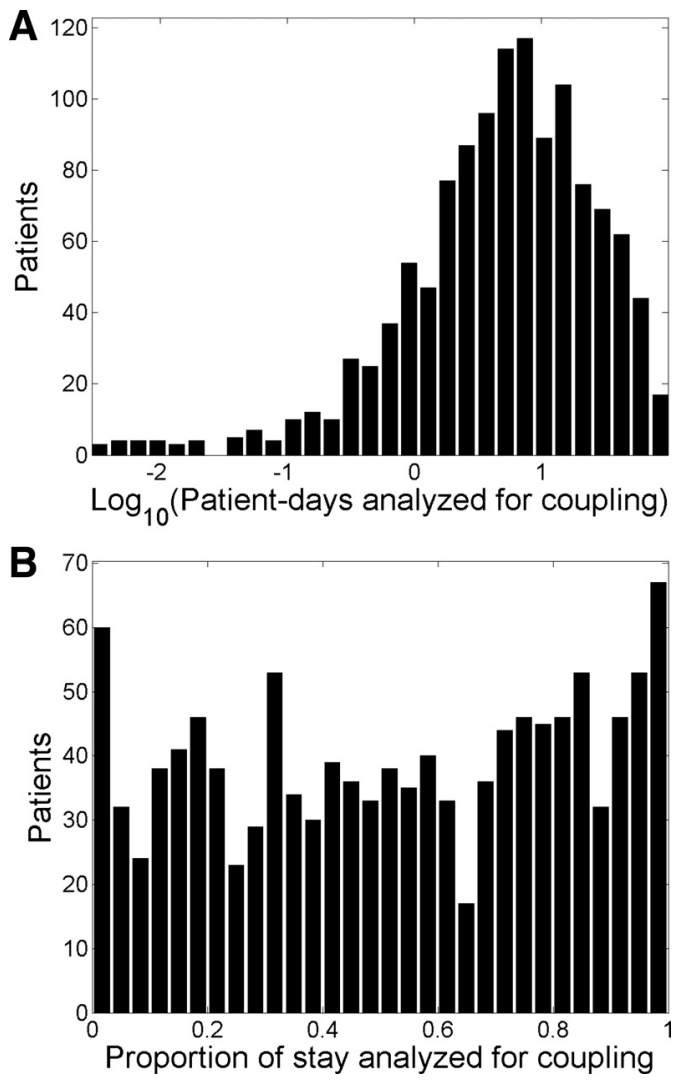

Fig. 3. Histogram of number of days $(A)$ and proportion of stay $(B)$ deemed suitable for analysis for patients from the UVA NICU.

cates that this measure of heartbeat synchronization to respiration is distinct from conventional heart rate variability metrics.

The probability density represented by the solid line in Fig. $2 C$ showed dependence of heartbeat timing on respiration, whereas the probability density represented by the broken line did not. We quantified the difference using the Shannon entropy. The probability densities shown as solid and broken lines in Fig. $2 C$ have Shannon entropies of $\mathrm{S}=1.611$ and $\mathrm{S}=$ 1.787 , respectively, where the maximum possible entropy is $\ln (2 \pi)$, or 1.84 .

Availability of data for analysis. Figure $3 A$ shows the histogram of number of days that met our criteria for signal quality and were analyzed for cardiorespiratory interaction in 1,202 patients. The mean number of records per patient was 34,600 , corresponding to an average of 13 days of data per patient. Figure $3 B$ shows the histogram of proportion of each patient's stay that met our criteria for signal quality and was analyzed for cardiorespiratory interaction. On average, we analyzed cardiorespiratory interaction in 52\% of each patient's stay.

Non-randomness of the probability densities. Figure $4 A$ shows the histogram of Shannon entropy of the probability densities (for example, shown in Fig. 2C) resulting from a series of random numbers in 1.7 million trials. We expect a uniform distribution of heartbeats in respiratory phase to simulate uncoupled cardiorespiratory dynamics. The entropies are shown as a function of the number of heartbeats in the trials. The $0.1 \%$ value for each heartbeat count is shown as a solid line. Thus, at each heartbeat count, $99.9 \%$ of entropy values from random trials were above this curve.

Figure $4 B$ shows the histogram of actual entropy and heartbeat count values for 1.7 million 4-min records from the NICU dataset. The circles represent the examples given in Fig. 2, A and $B$. Since points under the solid line have only $0.1 \%$ chance of occurring from a random process, we classified them as coupling of heartbeats and breaths. We find that $25.7 \%$ of measurements show coupling, i.e., have a $<0.1 \%$ chance of arising from random numbers.

Mechanism of cardiorespiratory interaction. We calculated cardioventilatory coupling in each record using the methods of Tzeng et al. (52) to investigate the relation to our measure of cardiorespiratory interaction. We calculated the Shannon entropy of histograms of the time between inhale and the previous R-wave, and classified records whose entropy had a $<5 \%$ chance of occurring from noise given the number of intervals as exhibiting cardioventilatory coupling (52). We found only moderate agreement between the two measures (Cohen's kappa statistic $\kappa=0.33$ ).

To investigate the relationship between RSA and cardiorespiratory interaction, we investigated the preference for heartbeats during inhale or exhale. Figure 5 shows the probability density for the fraction of heartbeats during inhale for all records from VLBW infants at times with no respiratory support and where cardiorespiratory interaction was evident. The fraction of heartbeats during inhale was determined by integrating the right half of the probability density of heartbeats as a function of respiratory phase. This process shifts the mode
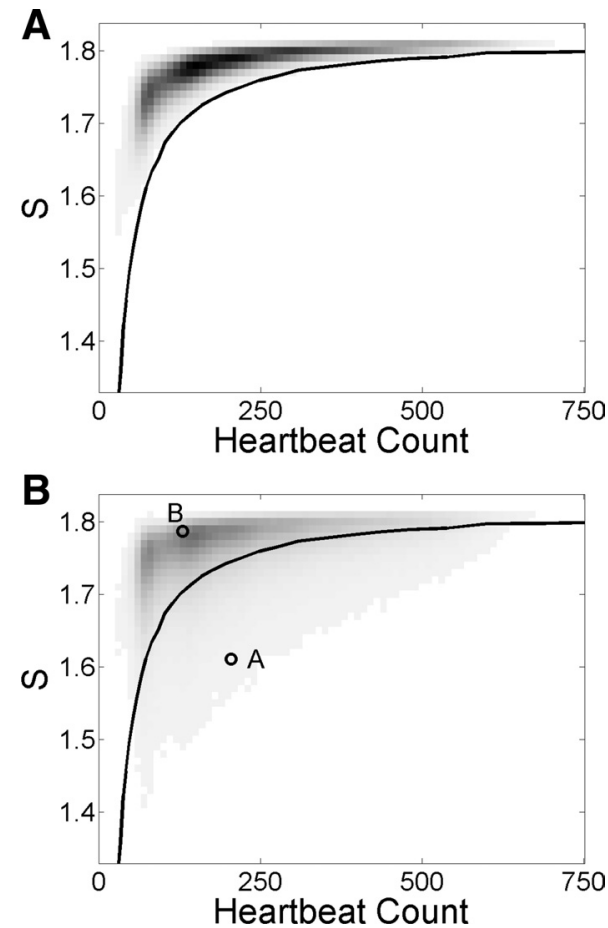

Fig. 4. Histogram of Shannon entropy of the probability density of heartbeats as a function of respiratory phase. Values are shown as a function of the number of heart beats in the window. A: histogram for 1.7 million trials using random numbers. $B$ : histogram for 1.7 million experimental measurements from 1,202 NICU patients. The lines are the value of the $0.1 \%$ measure of entropy for time series of random numbers. The two labeled circles are the results from Fig. 2. 


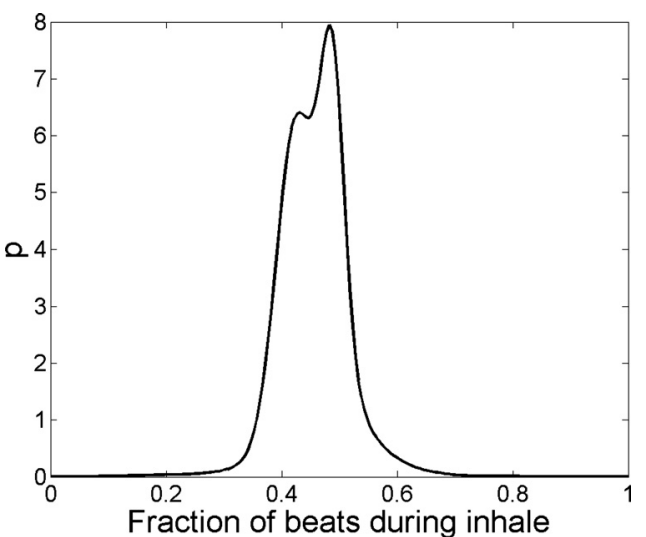

Fig. 5. Probability density function for the fraction of heartbeats during inhale. Results are from all records of VLBW patients at times with no respiratory support when cardiorespiratory interaction is evident. The density function has a bimodal distribution.

of the distribution in Fig. 5 toward fewer beats during inhale due to stretching of exhale. The distribution in Fig. 5 is bimodal with a second peak in the distribution at 0.43 ; this indicates preferential beating of the heart during exhale. Respiratory sinus arrhythmia, on the contrary, induces a high heart rate during inhale and should elicit preferential beating of the heart during inhale.

The degree of cardiorespiratory interaction was a function of PMA, but not of birth weight or gestational age at birth. Figure 6 shows cardiorespiratory interaction as a function of postmenstrual age (PMA), the sum of EGA at birth and time since birth. Non-VLBW infants were included in the analysis regardless of their ventilatory status. VLBW infants were included in the analysis only when they were extubated.

Figure $6 A$ shows the distribution of patients analyzed: nonVLBW infants (filled bars), extubated VLBW infants (open bars), and their union.

Figure $6 B$ shows cardiorespiratory interaction as a function of PMA for non-VLBW infants (black lines) and extubated VLBW infants (gray lines). Solid lines represent the mean, and broken lines represent the 95\% confidence interval. Cardiorespiratory interaction increased with PMA in both populations. There was, in fact, no statistically significant difference in cardiorespiratory interaction between non-VLBW patients and extubated VLBW patients after 32 wk PMA as tested by signed rank tests.

Figure $6 C$ shows cardiorespiratory interaction as a function of PMA for infants with estimated gestational ages (EGA) of $<32 \mathrm{wk}$ at birth (gray lines) and EGA of $\geq 32 \mathrm{wk}$ at birth (black lines). Cardiorespiratory interaction increased postnatally in both groups. In fact, between 32 and 38 wk PMA, cardiorespiratory interaction for the two groups was essentially identical. The falling number of VLBW infants after 38 wk made comparison more difficult. This analysis was based on EGA at birth and PMA, and did not consider birth weight or respiratory support. Thus each group has extubated VLBW infants and non-VLBW infants whose respiratory support was not known.

Clinical utility: safe discharge from the NICU. Figure 7 shows cardiorespiratory interaction as a function of time relative to discharge home without respiratory support for 148 extubated VLBW infants. The median prevalence of cardiorespiratory interaction in the previous $72 \mathrm{~h}$ was calculated for each infant each hour. Values are mean cardiorespiratory interaction for all infants each hour. The shaded region is the $95 \%$ confidence interval around the mean. The prevalence of cardiorespiratory interaction increased over the $7 \mathrm{wk}$ before discharge. A signed rank test between values 30 days and 10 days before safe discharge home (broken lines) indicates the medians at these points were significantly different $(P<0.001)$.

\section{DISCUSSION}

We studied cardiorespiratory interaction in 1,202 NICU infants using a breath-by-breath measure of cardiorespiratory interaction originally presented by Schäfer and coworkers (46).


Fig. 6. A: stacked histogram of the number of extubated VLBW infants (open bars), non-VLBW infants (filled bars), and their union as a function of PMA. $B$ : cardiorespiratory interaction as a function of PMA for non-VLBW infants (black lines) and extubated VLBW infants (gray lines). The heavy lines are the mean, and the thin lines are $95 \%$ confidence intervals. $C$ : cardiorespiratory interaction as a function of PMA for patients with EGA at birth of $<32$ wk (black lines) and those with EGA at birth of $\geq 32 \mathrm{wk}$ (gray lines). Data include both intubated and extubated babies. 


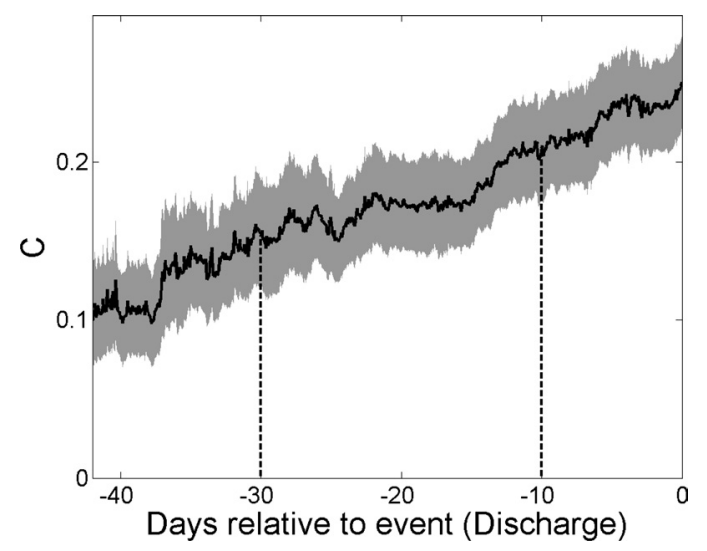

Fig. 7. Cardiorespiratory interaction in the absence of mechanical ventilation as a function of time until discharge to home without respiratory support in 148 VLBW infants. The median cardiorespiratory interaction in the prior $72 \mathrm{~h}$ was calculated for each patient each hour. The plot shows the median value for all patients each hour. The difference in values 30 and 10 days before discharge (broken lines) is highly significant using a signed rank test $(P=0.001)$.

Our major finding was that cardiorespiratory interaction increased with postnatal age, as measured from $26 \mathrm{wk}$ to approximately full-term gestation. Surprisingly, this relationship was independent of both the birth weight and the gestational age at birth. This indicates that development of cardiorespiratory interaction through the central and autonomic nervous systems may be insensitive to environmental influences. We also find that prevalence of interaction steadily increased during the 7 wk before the attending physician's decision to discharge the baby to home without respiratory support or cardiorespiratory monitoring.

The strength of our analytical approach was that each breath and heartbeat was identified individually in determining the respiratory phase at which each heartbeat fell. This eliminated respiratory and heart rate variability as confounders. Additionally, R-R intervals were not required by this measure, making it robust to missing beats, and the phase-based measure is not subject to cardiac aliasing, a common issue in frequency domain analysis of neonatal heart rate $(45,53)$. Finally, all signals were collected from conventional bedside monitors that are routinely operational throughout the hospitalization of all high-risk neonates, without the requirement of additional interfaces or technical attendance.

One limitation is that we did not collect respiratory support information for non-VLBW infants. For VLBW infants, we excluded times of mechanical ventilation since our ultimate goal was to find a reliable indicator of cardiorespiratory stability that could be used to help the clinician decide on the timing of a safe apnea-free discharge of the spontaneously breathing baby born prematurely. Another limitation is the requirement for very high-quality bedside monitoring (4). The usual reason for exclusion was noise in the chest impedance signal. We acquired 61 infant-years of data and found $18 \mathrm{yr}$ that had suitable chest impedance for reliable breath detection. We found $3.3 \mathrm{yr}$ of cardiorespiratory interaction, $\sim 18 \%$ of the analyzed time.

This is comparable with the original finding by Schäfer et al. of $\sim 30$ min of cardiorespiratory interaction in $4 \mathrm{~h}$ of recording (46). Other signals such as air flow measured by nasal thermistor or strain-gauge-based chest belts might increase propor- tion of time where accurate cardiorespiratory interaction might be measured, although long periods of recording many babies under such conditions would be impractical in the usual NICU environment. Finally, we note that one possible explanation of our results is that cardiorespiratory interaction is a surrogate for quiet sleep $(16,20,21)$. This is particularly relevant in the context of CVC, which is only observed in resting or sedated subjects $(16,51)$. A rigorous comparison with continuous sleep measures would be necessary to quantify this relationship.

We quantified cardiorespiratory interaction by the temporal association of heartbeats within the respiratory cycle. The measurement does not allow distinction, though, between the scenarios in which breathing dictates the timing of heartbeats (RSA) or the opposite scenario in which heartbeats dictate the timing of breaths (CVC). Schäfer et al. find that the prevalence of cardiorespiratory synchronization is diminished in patients with significant RSA (46), and Galletly and Larsen find this to be the case with CVC as well (15). Coupling in this context is mediated by baroreceptor inputs to the central respiratory pattern generator (52) and is only found in patients under anesthesia or in resting states $(16,51)$. We find only moderate agreement between the temporal association of heartbeats within the respiratory cycle and CVC. We also find no direct evidence for RSA in our dataset of neonatal intensive care patients. These findings indicate that multiple mechanisms contribute to our measure of cardiorespiratory interaction, and those mechanisms are uncertain. Future studies should be designed to investigate the underlying physiology of cardiorespiratory interaction by analyzing, e.g., variations in the probability density of heartbeats as a function of respiratory phase with PMA.

Regardless of the underlying coupling mechanisms, our findings demonstrate that cardiorespiratory interaction coincides with development of brain stem maturity in preterm infants. The prevalence of apnea of prematurity in neonatal intensive care patients declines with increasing PMA (6). This indicates improving central respiratory control in the ventrolateral medulla. A subset of cardiac motoneurons cohabit this region and develop interconnectivity with the neurons in the ventral respiratory column (49). Neural connections between these groups result in dependence of inter-heartbeat intervals on phase of respiration at which the beat occurred (12). Therefore, development of cardiorespiratory interaction should reflect brain stem development, whether mediated by baroreceptor afferents or sleep architecture in the case of CVC (16, 52 ), or by chemosensory pathways, the baroreflex loop, or respiratory gating in the case of RSA $(8,9,11,26,27)$.

Future studies will be required to understand the link of coupling to other measures of brain stem maturity, such as independent thermoregulation, coordination of sucking and breathing, and decreasing incidence of apnea. Moreover, we hypothesize that providing measures of cardiorespiratory interaction to critical care professionals will lead to safer and earlier discharge of prematurely born infants from intensive care. Continuous monitoring of premature infants using advanced mathematical approaches $(2,14,29,31)$ can also improve outcomes by identifying impending illness early, thus affording the clinician the ability to intervene sooner. This capability has been demonstrated in the large randomized trial of heart rate characteristics monitoring for early detection of neonatal sepsis (36). Since coupling of organs is correlated 



Fig. A1. A: time series of artificial EKG with no noise. B: Hilbert space of artificial EKG with no noise. The broken line is a segment of the time series shifted from the data for clarity. C: time series of artificial EKG with $60 \%$ noise. $D$ : Hilbert space of artificial EKG with $60 \%$ noise. $E$ : two-dimensional sample entropy as a function of percent noise.

with good health (17), continuously measuring cardiorespiratory interaction may provide early detection of subacute, potentially catastrophic illness. Future studies should test the hypothesis that falling cardiorespiratory interaction precedes clinically evident deterioration.

\section{APPENDIX}

A sine wave was used to simulate noise-free chest impedance. An EKG template was created by averaging 200 heartbeats and the noise-free EKG signal created by replicating the template at intervals from a clinically measured series. We created clinically appropriate noise for EKG and chest impedance by randomly selecting 10-min measured data segments and averaging the Fourier transforms. Additional Fourier transforms were averaged until the sum squared error between the power distribution for $n$ and $(n+1)$ segments was $<10^{-6}$. This required 613 windows for chest impedance and 882 for EKG. The noise time series was recovered using an inverse Fourier transform and normalized to unit variance.

Noise was added to the artificial time series in increasing amounts

$$
Y=\left(1-\sigma_{\text {noise }}\right) \alpha+\sigma_{\text {noise }} \xi
$$

Here $\alpha$ is a noise-free waveform with unit variance, $\xi$ is clinically appropriate noise time series with unit variance, and $\sigma_{\text {noise }}$ is the fraction of variance due to noise. The time series $Y$ has unit variance. Signal quality measures were then calculated at each value of $\sigma_{\text {noise }}$ to create a mapping between the signal quality measure and actual noise level.

Waveform analysis: EKG noise quantification. The noise in each EKG time series was quantified using sample entropy estimates (32, $43,44)$ extended into two dimensions. Sample entropy is the negative natural logarithm of the conditional probability that two segments from the time series with length $m+1$ will match given that the first $m$ points match. A match occurs when the maximum distance between two segments is less than a threshold $r$. We choose to define matches within a square rather than a circle to minimize computational requirements. High entropy of the EKG time series was interpreted as increased noise. Fig. A1, $A$ and $C$, shows EKG time series with 0 and $60 \%$ noise added, respectively, and Fig. A1, $B$ and $D$, shows the Hilbert space of the corresponding time series. The ordinate in Hilbert space is EKG voltage, and the abscissa is the Hilbert transform of the EKG. The Hilbert transform of a signal is

$$
H(y)=\frac{1}{\pi} P \int_{-\infty}^{\infty} \frac{f(x)}{x-y} d x
$$

Here $P$ indicates the Cauchy principal value of the integral. The Hilbert transform of a signal is the original signal shifted $90^{\circ}$ in phase (41). A sample segment of six points from the time series is shown as a broken line in Fig. A1B, shifted left for clarity. Sample entropy as a function of noise level is shown in Fig. A $1 E$ for $m=1$ and $r=0.4 \sigma$, where $\sigma$ is median absolute difference of the EKG time series.

Waveform analysis: chest impedance noise quantification. To quantify the noise in the chest impedance waveform, the power spectrum was calculated from the magnitude of a Fourier series. The power spectrum was normalized to unit area and detrended to remove the $1 / f$ noise component. This detrending was accomplished by fitting a line to the $\log$ frequency and $\log$ power in the decade between 1 and $10 \mathrm{~Hz}$, and subtracting the result from the power spectrum. The resulting spectrum consists of a peak corresponding to respiration and residual noise. By normalizing the spectrum to unit area, each ordinate corresponds to the variance at the corresponding abscissa. We therefore considered the largest ordinate to be the fraction of the time series variance corresponding to respiration. One minus the ordinate corresponds to the fraction of total variance corresponding to noise.

Fig. A2A shows a sine wave mimicking the chest impedance, and Fig. A2C shows the effect of adding $70 \%$ noise with clinically appropriate frequency content, keeping the variance equal to 1. Fig. A2, $B$ and $D$, shows the corresponding normalized power spectra after
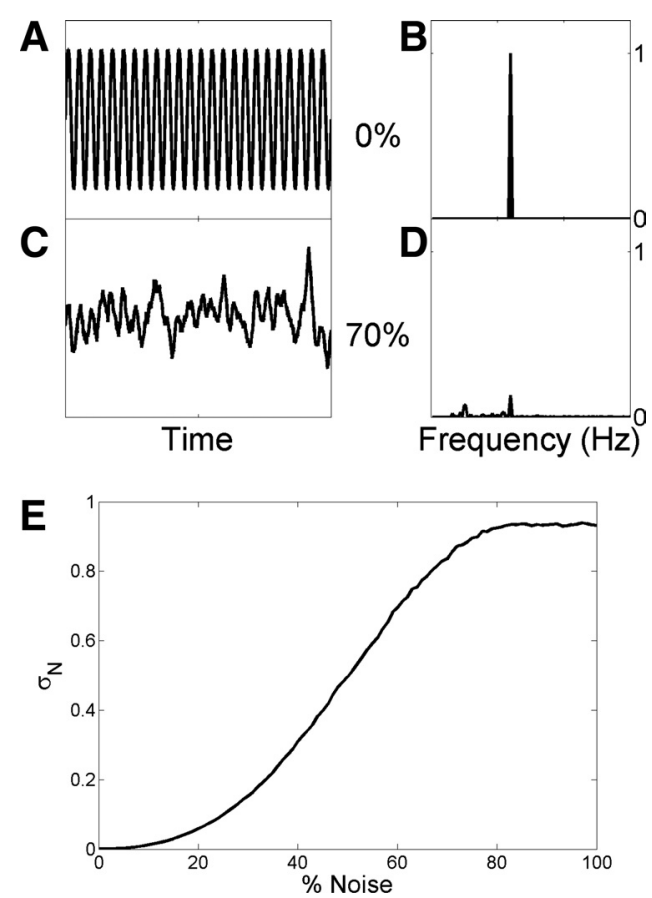

Fig. A2. A: time series of artificial chest impedance with no noise. $B$ : normalized and detrended power spectrum for artificial chest impedance with no noise. $C$ : time series of artificial chest impedance with $70 \%$ noise. $D$ : normalized and detrended power spectrum for artificial chest impedance with $70 \%$ noise. E: estimated fraction of time series variance attributed to noise as a function of noise percent for a sinusoid plus clinically appropriate noise. 
detrending to remove the $1 / f$ noise component. Increasing noise distributes power across a broad frequency range and decreases the fraction of total variance due to respiration. Fig. A2E shows the mapping between the estimated fraction of time series variance due to noise and for a sinusoid plus clinically appropriate noise. The estimated fraction of time series variance due to noise saturates at low noise due to the detrending and at high noise due to residual noise in the detrended spectrum (see Fig. A2D).

\section{GRANTS}

This work was funded by National Institute of Child Health and Human Development Grant 1 1RC2 HD-064488-02.

\section{DISCLOSURES}

Craig G. Rusin is CTO of Medical Informatics.

\section{AUTHOR CONTRIBUTIONS}

Author contributions: M.T.C., C.G.R., J.L.H., J.B.D., J.K., D.E.L., and J.R.M. conception and design of research; M.T.C. performed experiments; M.T.C., H.L., L.E.G., and D.E.L. analyzed data; M.T.C., C.G.R., J.L.H., J.B.D., B.D.V., A.P.-B., J.K., D.E.L., and J.R.M. interpreted results of experiments; M.T.C. prepared figures; M.T.C. and J.R.M. drafted the manuscript; M.T.C., J.L.H., J.B.D., J.K., D.E.L., and J.R.M. edited and revised the manuscript; M.T.C., C.G.R., J.L.H., H.L., J.B.D., L.E.G., B.D.V., A.P.-B., J.K., D.E.L., and J.R.M. approved the final version of the manuscript.

\section{REFERENCES}

1. Buchman TG, Stein PK, Goldstein B. Heart rate variability in critical illness and critical care. Curr Opin Crit Care 8: 311-315, 2002.

2. Cao H, Lake DE, Griffin MP, Moorman JR. Increased nonstationarity of neonatal heart rate before the clinical diagnosis of sepsis. Ann Biomed Eng 32: 233-244, 2004.

3. Chang KL, Monahan KJ, Griffin MP, Lake D, Moorman JR. Comparison and clinical application of frequency domain methods in analysis of neonatal heart rate time series. Ann Biomed Eng 29: 764-774, 2001.

4. Clifford GD, Long WJ, Moody GB, Szolovits P. Robust parameter extraction for decision support using multimodal intensive care data. Philos Transact A Math Phys Eng Sci 367: 411-429, 2009.

5. Darnall RA. ALTEs: still a puzzle after all these years. J Pediatr 154: 317-319, 2009.

6. Darnall RA, Ariagno RL, Kinney HC. The late preterm infant and the control of breathing, sleep, and brainstem development: A review. Clin Perinatol 33: 883-+, 2006.

7. Darnall RA, Kattwinkel J, Nattie C, Robinson M. Margin of safety for discharge after apnea in preterm infants. Pediatrics 100: 795-801, 1997.

8. Dean JB, Nattie EE. Central $\mathrm{CO}_{2}$ chemoreception in cardiorespiratory control. J Appl Physiol 108: 976-978, 2010.

9. Eckberg DL. The human respiratory gate. J Physiol 548: 339-352, 2003.

10. Eckberg DL. Human sinus arrhythmia as an index of vagal cardiac outflow. J Appl Physiol 54: 961-966, 1983.

11. Eckberg DL. Point:counterpoint: respiratory sinus arrhythmia is due to a central mechanism vs. respiratory sinus arrhythmia is due to the baroreflex mechanism. J Appl Physiol 106: 1740-1742; discussion 1744, 2009.

12. Eckberg DL, Kifle YT, Roberts VL. Phase relationship between normal human respiration and baroreflex responsiveness. J Physiol 304: 489-502, 1980.

13. Eichenwald EC, Aina A, Stark AR. Apnea frequently persists beyond term gestation in infants delivered at 24 to 28 weeks. Pediatrics 100: 354-359, 1997.

14. Flower AA, Moorman JR, Lake DE, Delos JB. Dynamical theory of periodic heart rate decelerations in premature infants. Exp Med Biol 235: 531-538, 2010.

15. Galletly DC, Larsen PD. Cardioventilatory coupling during anaesthesia. Br J Anaesth 79: 35-40, 1997.

16. Galletly DC, Larsen PD. Coupling of spontaneous ventilation to heart beat during benzodiazepine sedation. Br J Anaesth 78: 100-101, 1997.

17. Godin PJ, Buchman TG. Uncoupling of biological oscillators: a complementary hypothesis concerning the pathogenesis of multiple organ dysfunction syndrome. Crit Care Med 24: 1107-1116, 1996.

18. Grossman P. Respiratory and cardiac rhythms as windows to central and autonomic biobehavioral regulation: selection of window frames, keeping the panes clean and viewing the neural topography. Biol Psychol 34: 131-161, 1992.

19. Gustafson KM, Allen JJ, Yeh HW, May LE. Characterization of the fetal diaphragmatic magnetomyogram and the effect of breathing movements on cardiac metrics of rate and variability. Early Hum Dev 87: 467-475, 2011.

20. Harper RM, Walter DO, Leake B, Hoffman HJ, Sieck GC, Sterman MB, Hoppenbrouwers T, Hodgman J. Development of sinus arrhythmia during sleeping and waking states in normal infants. Sleep 1: 33-48, 1978.

21. Hathorn MK. Analysis of periodic changes in ventilation in new-born infants. J Physiol 285: 85-99, 1978.

22. Hathorn MK. The rate and depth of breathing in new-born infants in different sleep states. J Physiol 243: 101-113, 1974.

23. Hathorn MK. Respiratory sinus arrhythmia in new-born infants. J Physiol 385: 1-12, 1987.

24. Hundley BW, Sica AL, Gootman PM. Rhythmicities in sympathetic discharge: a signal of cardiorespiratory integration in developing animals. Ann NY Acad Sci 940: 416-430, 2001.

25. Indic P, Bloch-Salisbury E, Bednarek F, Brown EN, Paydarfar D, Barbieri R. Assessment of cardio-respiratory interactions in preterm infants by bivariate autoregressive modeling and surrogate data analysis. Early Hum Dev 87: 477-487, 1016.

26. Karemaker JM. Counterpoint: Respiratory sinus arrhythmia is due to the baroreflex mechanism. J Appl Physiol 106: 1742-1743; discussion 1744, 2009.

27. Katona PG, Jih F. Respiratory sinus arrhythmia: noninvasive measure of parasympathetic cardiac control. J Appl Physiol 39: 801-805, 1975.

28. Kattwinkel J, Brooks J, Keenan ME, Malloy M. Infant sleep position and sudden infant death syndrome (SIDS) in the United States: joint commentary from the American Academy of Pediatrics and selected agencies of the Federal Government. Pediatrics 93: 820, 1994.

29. Kovatchev BP, Farhy LS, Cao H, Griffin MP, Lake DE, Moorman JR. Sample asymmetry analysis of heart rate characteristics with application to neonatal sepsis and systemic inflammatory response syndrome. Pediatr Res 54: 892-898, 2003.

30. Kralemann B, Cimponeriu L, Rosenblum M, Pikovsky A, Mrowka R. Phase dynamics of coupled oscillators reconstructed from data. Phys Rev E Stat Nonlin Soft Matter Phys 77: 066205, 2008.

31. Lake DE, Richman JS, Griffin MP, Moorman JR. Sample entropy analysis of neonatal heart rate variability. Am J Physiol Regul Integr Comp Physiol 283: R789-R797, 2002.

33. Larsen PD, Tzeng YC, Sin PYW, Galletly DC. Respiratory sinus arrhythmia in conscious humans during spontaneous respiration. Respir Physiol Neurobiol 174: 111-118, 2010.

34. Lee H, Rusin CG, Lake DE, Clark MT, Guin LE, Smoot TJ, PagetBrown AO, Vergales BD, Kattwinkel J, Moorman JR, Delos JB. A new algorithm for detecting central apnea in neonates. Physiol Meas 33: $1-17,2012$.

35. Li Q, Mark RG, Clifford GD. Robust heart rate estimation from multiple asynchronous noisy sources using signal quality indices and a Kalman filter. Physiol Meas 29: 15-32, 2008.

36. Moorman JR, Carlo WA, Kattwinkel J, Schelonka RL, Porcelli PJ, Naverrete CT, Bancalari E, Aschner JL, Walker MW, Perez JA, Palmer C, Wagner DP, Stukenborg GJ, Lake DE, O'Shea TM. Mortality reduction by heart rate characteristics monitoring in very low birthweight neonates: a randomized trial. J Pediatr 159: 900-906, 2011.

37. Morris JA Jr, Norris PR, Ozdas A, Waitman LR, Harrell FE Jr, Williams AE, Cao H, Jenkins JM. Reduced heart rate variability: an indicator of cardiac uncoupling and diminished physiologic reserve in 1,425 trauma patients. J Trauma 60: 1165-1173; discussion 1173-1164, 2006.

38. Mrowka R, Cimponeriu L, Patzak A, Rosenblum MG. Directionality of coupling of physiological subsystems: age-related changes of cardiorespiratory interaction during different sleep stages in babies. Am J Physiol Regul Integr Comp Physiol 285: R1395-R1401, 2003.

39. Norris PR, Ozdas A, Cao H, Williams AE, Harrell FE, Jenkins JM, Morris JA Jr, Waitman LR, Harrell FE Jr. Cardiac uncoupling and heart rate variability stratify ICU patients by mortality: a study of 2088 trauma patients. Ann Surg 243: 804-812; discussion 812-804, 2006.

40. Pan J, Tompkins WJ. A real-time QRS detection algorithm. IEEE Trans Biomed Eng 32: 230-236, 1985. 
41. Pikovsky A, Rosenblum M, Kurths J. Synchronization: A Universal Concept in Nonlinear Sciences. Cambridge: Cambridge Univ. Press, 2001.

42. Porges SW, Grossman P, Janssen KH, Vaitl D. Respiratory sinus arrhythmia: physiological basis, quantitative methods, and clinical implications. In: Cardiorespiratory and Cardiosomatic Psychophysiology. New York: Plenum, 1986, p. 101-106.

43. Richman JS, Lake DE, Moorman JR. Sample entropy. Methods Enzymol 384: 172-184, 2004.

44. Richman JS, Moorman JR. Physiological time series analysis using approximate entropy and sample entropy. Am J Physiol Heart Circ Physiol 278: H2039-H2049, 2000.

45. Rother M, Witte H, Zwiener U, Eiselt M, Fischer P. Cardiac aliasing: a possible cause for the misinterpretation of cardiorespirographic data in neonates. Early Hum Dev 20: 1-12, 1989.

46. Schäfer C, Rosenblum MG, Kurths J, Abel HH. Heartbeat synchronized with ventilation. Nature 392: 239-240, 1998.
48. Shannon CE. A mathematical theory of communication. Bell System Tech $J$ 27: 379-423, 1948.

49. Spyer KM, Gourine AV. Chemosensory pathways in the brainstem controlling cardiorespiratory activity. Philos Trans $R$ Soc Lond B Biol Sci 364: 2603-2610, 2009.

50. Tarassenko L, Clifford G, Townsend N. Detection of ectopic beats in the electrocardiogram using an auto-associative neural network. Neural Process Lett 14: 15-25, 2001.

51. Tzeng YC, Larsen PD, Galletly DC. Cardioventilatory coupling in resting human subjects. Exp Physiol 88: 775-782, 2003.

52. Tzeng YC, Larsen PD, Galletly DC. Mechanism of cardioventilatory coupling: insights from cardiac pacing, vagotomy, and sinoaortic denervation in the anesthetized rat. Am J Physiol Heart Circ Physiol 292: H1967-H1977, 2007.

53. Witte H, Zwiener U, Rother M, Glaser S. Evidence of a previously undescribed form of respiratory sinus arrhythmia (RSA): the physiological manifestation of "cardiac aliasing". Pflügers Arch 412: 442-444, 1988.

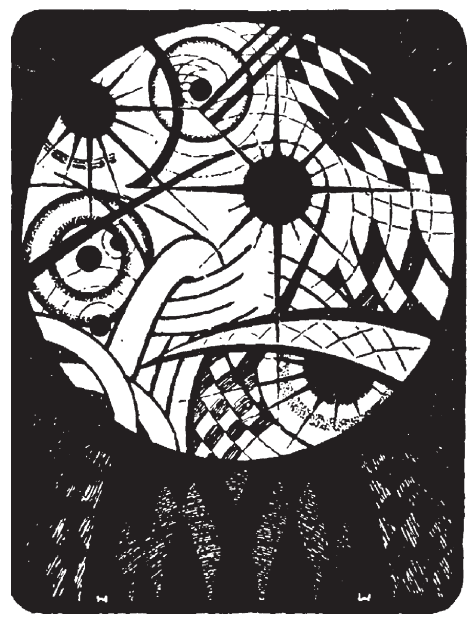

